\title{
How to differenti-8 Gleason scores?
}

Although well established, many prostate cancer experts argue that Gleason score is not the best method to stratify prostate cancer. A new five-tiered stratification system recommended by the International Society of Urological Pathology is thought to be more accurate; however, it still puts Gleason 8 tumours into one prognostic group, despite heterogeneity in outcomes observed between Gleason 3+5, 5+3, and $4+4$ cancers. Newly published data have now lent further weight to this argument, showing that Gleason score $3+5,5+3$, and $4+4$ cancers have different prognoses, despite all being considered one entity-Gleason score 8-in current clinical practice.

The study, published in European Urology, used a prospectively assembled database to delineate the differences in prostate-cancer-specific mortality (PCSM) and all-cause mortality (ACM) across the three subgroups of Gleason 8 . 462 men with a median age of 72 years were included in the study, all of whom had at least one biopsy core displaying Gleason 8 characteristics. After a median follow-up duration of 7.6 years, 118 men in the cohort had died, 26 of prostate cancer. Risk of PCSM and ACM were significantly higher in the combined Gleason $3+5 / 5+3$ group than in the $4+4$ group, even after adjusting for age, treatment received, and a treatment propensity score. After splitting the $3+5 / 5+3$ cohort into its component groups, the higher PCSM and ACM risks remained, but were only significant for Gleason $5+3$ compared with $4+4$, suggesting that the presence of Gleason 5, and the percentage thereof, itself provides prognostic data, and that this information should be considered in studies that currently stratify tumours as merely Gleason 8.

Annette Fenner

Original article Huynh, M. A. et al. Gleason score $3+5$ or $5+3$ versus $4+4$ prostate cancer: the risk of death. Eur. Urol. doi:10.1016/j.eururo.2015.08.05 